\title{
A Special Class of Stationary Flows for Two-Dimensional Euler Equations : A Statistical Mechanics Description
}

\author{
E. Caglioti $^{1}$, P. L. Lions ${ }^{2}$, C. Marchioro ${ }^{3}$, and M. Pulvirenti ${ }^{4}$ \\ 1 Dipartimento di Fisica, Università di Roma "La Sapienza", Roma, Italy \\ 2 Ceremade, Université Paris-Dauphine, Paris, France \\ 3 Dipartimento di Matematica, Universitá di Roma "La Sapienza", Roma, Italy \\ 4 Dipartimento di Matematica, Universitá di L’Aquila, Italy
}

Received November 6, 1990; in revised form July 16, 1991

Abstract. We consider the canonical Gibbs measure associated to a $N$-vortex system in a bounded domain $\Lambda$, at inverse temperature $\widetilde{\beta}$ and prove that, in the limit $N \rightarrow \infty, \widetilde{\beta} / N \rightarrow \beta, \alpha N \rightarrow 1$, where $\beta \in(-8 \pi,+\infty)$ (here $\alpha$ denotes the vorticity intensity of each vortex), the one particle distribution function $\varrho^{N}=\varrho^{N}(x), x \in \Lambda$ converges to a superposition of solutions $\varrho_{\beta}$ of the following Mean Field Equation:

$$
\left\{\begin{array}{l}
\varrho_{\beta}(x)=\frac{e^{-\beta \psi}}{\int_{\Lambda} e^{-\beta \psi}} ; \quad-\Delta \psi=\varrho_{\beta} \text { in } \Lambda \\
\left.\psi\right|_{\partial \Lambda}=0 .
\end{array}\right.
$$

Moreover, we study the variational principles associated to Eq. (A.1) and prove that, when $\beta \rightarrow-8 \pi^{+}$, either $\varrho_{\beta} \rightarrow \delta_{x_{0}}$ (weakly in the sense of measures) where $x_{0}$ denotes an equilibrium point of a single point vortex in $\Lambda$, or $\varrho_{\beta}$ converges to a smooth solution of (A.1) for $\beta=-8 \pi$. Examples of both possibilities are given, although we are not able to solve the alternative for a given $\Lambda$. Finally, we discuss a possible connection of the present analysis with the 2-D turbulence.

\section{Table of Contents}

1. Introduction . . . . . . . . . . . . . . . . 502

2. Formulation of the Statistical Mechanics Problem . . . . . . . . . . . . . 505

3. Estimates on the Correlation Functions . . . . . . . . . . . . . . . . . 508

4. Proof of the Convergence Theorem . . . . . . . . . . . . . . . . . . 513

5. Solutions of the Mean-Field Equation with Radial Symmetry . . . . . . . . . 513

6. The Mean Field Equation in $\mathbb{R}^{2}$. . . . . . . . . . . . . . . . . . . 514

7. Some Properties of Solutions of the Mean-Field Equations . . . . . . . . . . 516

* Work partially supported by CNR (GNFM) and MPI 


\section{Introduction}

A natural route to the investigation of the fully turbulent behavior of a real flow is the study of invariant measures for the Euler equation. This in analogy with the Classical Statistical Mechanics where the Gibbs measures, which are stationary with respect to the Newton evolution, take into account the configurations which are relevant for the thermodynamical behavior of the system.

In the easiest two-dimensional case it is possible to construct, according to the Gibbs prescription, a class of Gaussian measures formally invariant. However, such measures do not seem to be physically relevant in the study of stationary turbulence: the model is too naive since it describes only free fields and the spectra computed with this ensemble are very far from those observed experimentally and computed numerically $[1,2,6,11,20,27,36]$.

Another approach is that proposed by Onsager. The basic idea is simple and natural. One introduces a finite dimensional Hamiltonian system, called the vortex model, which describes in some sense, the Euler flows whenever the vorticity field is a linear combination of $\delta$-functions concentrated in points of the physical space [33]. Thus, one can consider the Statistical Mechanics of such point vortices and the Gibbs measures associated to such a system can be considered as invariant measures for the Euler flows. The parameters characterizing these measures are the inverse temperature $\widetilde{\beta}$, the number of vortices $N$, and their vorticity intensities $\alpha_{1}, \ldots, \alpha_{N}$. If one believes in the possibility of having a universal theory of the stationary turbulence, namely not depending on the details of the system (in this case $N$ and $\left.\left\{\alpha_{i}\right\}_{1=1, \ldots, N}\right)$ one can try to investigate the limit as $N \rightarrow \infty, \alpha_{i} \rightarrow 0$ is such a way that the total vorticity remains bounded.

As noticed by Onsager, we are not interested really in the thermodynamical behavior of the system so that there is no reason to consider only positive $\widetilde{\beta}$ which we will assume to vary over the largest possible subset of $\mathbb{R}$.

Fröhlich and Ruelle proved that, in the standard thermodynamic limit of a neutral vortex gas, no negative temperature states exist [19]. However, other limits are conceivable.

We investigate here the above (Mean Field) limit and we find that the weak limits of the Gibbs measures, when $N \rightarrow \infty, \frac{\widetilde{\beta}}{N} \rightarrow \beta \in(-8 \pi,+\infty)$, do concentrate on very particular stationary solutions of the 2 -D Euler equation. Although we prove an absence of fluctuations in the above Mean Field limit, we believe that the stationary solutions we obtain in this way might play a role in understanding the 2-D turbulence as we shall discuss later on. Anyway, these solutions are interesting in themselves in particular since they satisfy a variational principle: they "minimize the energy-entropy functional."

The paper is organized as follows: we first formulate in Sect. 2 our problem. In Sect. 3 and 4 we study the Mean Field limit and we characterize the set of cluster points of the Gibbs measures for the point vortex system. Sections 5 and 6 introduce some (almost) explicit solutions in the case of a rotationally symmetric domain or in the case of the whole space $\mathbb{R}^{2}$. In Sect. 7, we develop a mathematical analysis of the Mean Field equation.

At this stage, it is worth making a remark on the turbulence problem. As noticed in [36], if one computes the energy spectrum via the canonical Gibbs measure for a point vortex system, one finds some unphysical part of order $k^{-1}$ which corresponds to the self-energy of each point vortex. This part disappears in a 
Mean Field type limit. On the other hand, when doing so, we loose fluctuations and this seems to indicate that the Gibbs measure might not be adequate to describe turbulent phenomena. However, numerical simulations $[7,8]$ show that the vortices have a tendency to create local clusters which have approximately a circular shape. We do not exclude that such clusters are indeed related to the mean field solutions we obtain and study.

More precisely, we believe that, due to the rotational invariance, the vortices likely arrange in clusters which look like the (negative temperature) solutions described in Sect. 6 . This is only a local description. The global measure, which we are not able to construct, could be a superposition of such solutions. This cannot be an invariant measure for the Euler flow, but we expect that, if suitably constructed, it should be practically invariant on relatively large scale of times.

We now discuss the mathematical aspects of our analysis. Our starting point is the canonical Gibbs measure associated to a system of $N$ vortices with intensity $\alpha=1 / N$ at inverse temperature $\hat{\beta}$ in a bounded domain $\Lambda$. We prove that such a measure exists only for $\widetilde{\beta} \in\left(-8 \pi / \alpha^{2} N,+\infty\right)$. In the limit $N \rightarrow \infty, \widetilde{\beta} \rightarrow \infty$, $\widetilde{\beta} / N \rightarrow \beta \in(-8 \pi,+\infty)$, we find that the typical configurations of the vortex system arrange themselves to form a special solution of the 2-D Euler equation (or a convex combination of them). Let us mention at this stage that we follow the approach of Messer and Spohn [31] with some additional difficulties due to the singularity of the point vortex interaction.

Let $\psi$ be the stream function associated to such solutions, then we show that $\psi$ must satisfy the following non-linear elliptic problem:

$$
\begin{aligned}
-\Delta \psi & =\frac{e^{-\beta \psi}}{\tilde{Z}}, \\
\tilde{Z} & =\int_{\Lambda} e^{-\beta \psi} d x, \\
\psi & =0 \quad \text { on } \partial \Lambda .
\end{aligned}
$$

Moreover the solution(s) chosen by the vortex system minimize for $\beta>0$, (respectively maximize for $\beta<0$ ) both the free energy functional

$$
\begin{aligned}
f(\psi)= & \frac{1}{2} \int_{\Lambda}|\nabla \psi|^{2}+\frac{1}{\beta} \int_{\Lambda} \omega \log \omega, \\
& \text { with the constraints } \omega \geqq 0, \int_{A} \omega=1,
\end{aligned}
$$

where $\omega=-\Delta \psi$ is the vorticity field, and maximize (this in fact equivalent)

$$
g(\psi)=-\frac{1}{\beta} \log \int_{A} e^{-\beta \psi}-\frac{1}{2} \int_{A}|\nabla \psi|^{2}
$$

which is obtained from $f$ by using (1.1).

The analysis of Eq. (1.1) or the variational problem associated to the functionals (1.2) and (1.3) is very different for $\beta \geqq 0$ and for $\beta \in(-8 \pi, 0)$. In the first case, existence and uniqueness for the solution of the problem is ensured [22]. The second case (namely $\beta<0$ ) is more delicate. Let us first recall that there have been many studies of Eq. (1.1) when $\tilde{Z}$ is a fixed parameter but the presence of such a (nonlinear) term changes drastically the nature of the problem. Since we are mainly interested in solutions of (1.1) which maximize (1.2) and (1.3), we first observe that 
the Mean Field limit yields in particular the existence of minima for $\beta>-8 \pi$ and we will show why this is the optimal bound. Indeed, by an inequality due to Moser [32] - see also Onofri [33], Hong [26] - one can recover the existence of such a maximizing solution for $\beta>-8 \pi$ and one also shows that $\sup f$ (or $\sup g$ ) becomes $+\infty$ when $\beta<-8 \pi$. We found rather striking the fact that a Statistical Mechanics argument allows to recover the optimal constraint in a subtle inequality for Sobolev spaces.

Letting $\beta$ go to $-8 \pi$ is a natural problem that we consider here. First of all, in the case of a ball, one knows by the results of Gidas, Ni, and Nirenberg [21], that all solutions are radially symmetric and as it is well-known, they can be computed explicitly. This is how we check that there is a unique solution of (1.1) for all $\beta>-8 \pi$ if $\Lambda$ is a ball and when $\beta \rightarrow-8 \pi$, this solution concentrates at 0 : more precisely $\omega=\frac{1}{Z} e^{-\beta \psi},|\nabla \psi|^{2}\|\nabla \psi\|^{-2}, \omega \log \omega\left(\int_{\Lambda} \omega \log \omega\right)^{-1}$ converge (weakly in the sense of measures) to $\delta_{0}$.

We are not able to determine whether this concentration phenomenon occurs for a given domain $\Lambda$. However, we prove that, as $\beta \rightarrow-8 \pi_{+}$, maxima of (1.2) either converge to a maximum of (1.2) or concentrate, in the above sense, at a point $x_{0} \in \Lambda$ which is a maximum point of $\gamma(x)=\frac{1}{2} \tilde{\gamma}(x, x)$, where $\tilde{\gamma}$ is the regular part of the Gren's function of the domain $\Lambda$. Furthermore, in the latter case, the behavior of $\omega$ near $x_{0}$ is then identical to the one of radial solutions concentrating at 0 (with an appropriate scaling). We prove these facts by a convenient adaptation of some arguments developed by Lions [28] (concentration-compactness method). Once, the concentration phenomenon is established, the fact that $x_{0}$ is a critical point of the regular part of the Green's function which is physically obvious follows, exactly as observed in Brézis and Peletier [10], Rey [37], Han [25] for somewhat related problems, from a general identity (related to translation invariance) due to Esteban and Lions [18].

It is not really surprising that such concentration phenomena take place in our problem since equations like (1.1) but formulated on $S^{2}$ do enjoy similar properties - see Hong [26], Bahri and Coron [5], Chang and Yang [12, 13], Chen and Ding [14], Han [24]. In some sense, this type of equation is the analogue of the so-called semilinear equations with the critical Sobolev exponents. For such problems and related ones, concentration phenomena have been studied by many authors - see Sacks and Uhlenbeck [39], Lions [28], Struwe [40]. However, one of the difficulties we encounter here is the dependence of, say, $\sup g(\psi)$ (for $\beta=-8 \pi)$ upon the geometry of $\Lambda$, dependence which forces in fact the concentration point to be a maximum point of the regular part of the Green's function and explains the above alternative (regular behavior or concentration). Let us also mention at this point that the regular part of the Green's function really appears in our argument as a "renormalized" functional deduced from $f$ when $\beta=-8 \pi$.

Let us also mention that there may exist solutions of (1.1) for some $\beta<-8 \pi$ or even for all $\beta$ in the case of the annulus: there, such solutions are not maxima of $f$ for $\beta<-8 \pi$. On the other hand, when $\Lambda$ is starshaped, Pohozaev's identity [35] yields the existence of a critical value $\beta_{c}$ such that no solution of (1.1) can exist if $\beta \leqq \beta_{c}\left(\beta_{c}=-8 \pi\right.$ when $\Lambda$ is a ball $)$.

Let us finally observe that possible concentrations have been studied for general solutions of two-dimensional incompressible Euler equations (timedependent flows and steady flows) in Di Perna and Majda $[15,16]$. The punctual nature of the concentration set in our special case is of course due to the special 
nature of Gibbs measures and exhibits an infinite energy limit while the possible concentrations in $[15,16]$ have bounded energy.

After this paper was submitted for publication, we were advised by M. Kiesling that he obtained independently results quite similar to those proven here in a preprint (submitted to Commun. Pure Appl. Math.).

\section{Formulation of the Statistical Mechanics Problem}

We consider a system of $N$ identical point vortices in a smooth, bounded, connected, open domain $\Lambda$ of $\mathbb{R}^{2}$. The Hamiltonian of the system is given by $\alpha^{2} U$, where

$$
U\left(x_{1}, \ldots, x_{N}\right)=\frac{1}{2} \sum_{i \neq j \geqq 1}^{N} V\left(x_{i}, x_{j}\right)+\sum_{i=1}^{N} \gamma\left(x_{i}\right),
$$

and where $\left(x_{1}, \ldots, x_{N}\right) \in \Lambda^{N}$ are the positions of the point vortices. Furthermore, $\alpha>0$ is the vorticity intensity (the same for all vortices) and $V(x, y)$ is the Green's function of the Poisson equation in $\Lambda$ with Dirichlet boundary conditions. As it is classical, we have on $\Lambda \times \Lambda$

$$
V(x, y)=-\frac{1}{2 \pi} \log |x-y|+\tilde{\gamma}(x, y)
$$

where $\tilde{\gamma}: \Lambda \times \Lambda \rightarrow \mathbb{R}$ is symmetric and harmonic in each variable. Finally we set

$$
\gamma(x)=\frac{1}{2} \tilde{\gamma}(x, x) \text { on } \Lambda \text {. }
$$
by

The canonical Gibbs measure associated to the above Hamiltonian is defined

$$
\mu^{\alpha, \tilde{\beta}, N}\left(d x_{1} \ldots d x_{N}\right)=Z_{\alpha, \tilde{\beta}}(N)^{-1} e^{-\tilde{\beta} \alpha^{2} U\left(x_{1}, \ldots, x_{N}\right)} d x_{1} \ldots d x_{N}
$$

where

$$
Z_{\alpha, \tilde{\beta}}(N)=\int_{\Lambda^{N}} e^{-\alpha^{2} \tilde{\beta} U} d x_{1} \ldots d x_{N}
$$

is the partition and $\widetilde{\beta}$ is proportional to the inverse temperature. Since we are interested in the measures (2.4) as invariant measures for the $N$-vortex system given by the Hamiltonian (2.1), we may and we shall consider positive as well as negative values of the parameter $\widetilde{\beta}$.

The following lemma yields the exact range of temperatures for which the measures (2.4) make sense and the integral in (2.5) converges.

Lemma 2.1. $Z_{\alpha, \tilde{\beta}}(N)<+\infty$ if and only if $\widetilde{\beta} \in\left(-\frac{8 \pi}{\alpha^{2} N}, \infty\right)$. Moreover, in this range of temperature, the following estimate holds:

$$
Z_{\alpha, \tilde{\beta}}(N) \leqq C(\widetilde{\beta} \alpha, N \alpha,|\Lambda|)^{N},
$$

where $C$ is a positive constant, depending only on the products $\widetilde{\beta} \alpha, N \alpha$, and on $|\Lambda| \equiv \operatorname{meas} \Lambda$.

Proof. Suppose $\widetilde{\beta}>0$. By the positivity of the interaction $V$,

$$
Z_{\alpha, \tilde{\beta}}(N) \leqq\left(\int_{\Lambda} d x e^{-\tilde{\beta} \alpha_{2} \gamma(x)}\right)^{N} .
$$


Since $\gamma(x)$ diverges logarithmically when $x \rightarrow \partial \Lambda$, estimate (2.6) is proven.

For $\widetilde{\beta}<0$ we have:

$Z_{\alpha, \tilde{\beta}}(N) \leqq \int d x_{1} \ldots d x_{N} \prod_{i=1}^{N} \prod_{\substack{j=1 \\ j \neq i}}^{N} e^{-\frac{\tilde{\beta} \alpha^{2}}{2} V\left(x_{i}, x_{j}\right)} \leqq \prod_{i=1}^{N}\left(\int d x_{1} \ldots d x_{N} \prod_{\substack{j=1 \\ j \neq i}}^{N} e^{-\frac{N \tilde{\beta} \alpha^{2}}{2} V\left(x_{i}, x_{j}\right)}\right)^{1 / N}$.

Since $V(x, y) \leqq-\frac{1}{2 \pi} \log |x-y|+c$, it follows that the above integral is bounded, provided that $-\widehat{\beta} \alpha^{2} N<8 \pi$. In this case estimate (2.6) follows easily.

On the other hand, we have

$$
\begin{aligned}
Z_{\alpha, \tilde{\beta}}(N) \geqq & \left(\int_{A_{\varepsilon}} d x_{1} \ldots d x_{N} \prod_{i=1}^{N} \prod_{\substack{j \neq i \\
j=1}}^{N}\left(\frac{1}{\left|x_{i}-x_{j}\right|}\right)^{-\tilde{\beta} \alpha^{2} / 4 \pi}\right) \\
& \times e^{-\tilde{\beta} \alpha^{2} m N} e^{\tilde{\beta} \alpha^{2} \frac{N(N-1) d}{2}},
\end{aligned}
$$

where

$$
A_{\varepsilon}=\left\{\left(x_{1}, \ldots, x_{N}\right)\left|x_{1} \in \Sigma_{R},\right| x_{1}-x_{i} \mid<\frac{\varepsilon}{2}, i=2 \ldots N\right\}
$$

and $\Sigma_{R}$ is the ball of radius $R$, centered at the origin (that we may always assume to be in $\Lambda)$ and $R<\frac{\operatorname{dist}(0, \partial \Lambda)}{2}$. Moreover, $m=\inf _{x \in \Sigma_{R}} \gamma(x)$ and $d>0$ is a suitable constant for which $V(x, y) \geqq-\frac{1}{2 \pi} \log |x-y|-d$.

The integral appearing in (2.9) is bounded from below by:

$$
\text { const }\left(\frac{\varepsilon}{2}\right)^{2(N-1)}\left(\frac{1}{\varepsilon}\right)^{-N(N-1) \cdot \frac{\tilde{\beta} \alpha^{2}}{4 \pi}}
$$

The above expression does not vanish in the limit $\varepsilon \rightarrow 0$ unless $\widehat{\beta}>-\frac{8 \pi}{\alpha^{2} N}$ and this completes the proof of Lemma 2.1.

We are interested in the asymptotic behavior of $\mu^{N, \tilde{\beta}, \alpha}$ in the limit $N \rightarrow \infty$ when $\alpha=\frac{1}{N}$ and $\widetilde{\beta}=\beta N$ with $\beta$ fixed. By Lemma 2.1, $\beta \in(-8 \pi,+\infty)$. For fixed $\beta$, the sequence of Gibbs measures $\mu^{N, \tilde{\beta}, \alpha}$ and the partition functions $Z_{\alpha, \tilde{\beta}}(N)$ depend only on $N$ and will be denoted by $\mu^{N}$ and $Z(N)$ respectively.

The family of correlation functions $\left\{\varrho_{j}^{N}\right\}_{j=1}^{N}$ (see [37]) are defined as follows:

$$
\varrho_{j}^{N}\left(x_{1}, \ldots, x_{j}\right)=\int d x_{j+1} \ldots d x_{N} \mu^{N}\left(x_{1} \ldots x_{N}\right)
$$

and corresponds to the probability density of finding the first $j$ particles in the positions $x_{1} \ldots x_{j}$. By an easy calculation:

$$
\varrho_{j}^{N}\left(X_{j}\right)=\frac{e^{-\frac{\beta}{N} U\left(x_{\jmath}\right)}}{Z(N)} \int d X_{N-j} e^{-\frac{\beta}{N} W\left(X_{J} \mid X_{N-\jmath}\right)} e^{-\frac{\beta}{N} U\left(X_{N-\jmath}\right)},
$$


where $X_{j}=\left\{x_{1} \ldots x_{j}\right\} X_{N-j}=\left\{x_{j+1} \ldots x_{N}\right\}$ and

$$
W\left(X_{j} \mid X_{N-j}\right)=\sum_{x \in X_{j}} \sum_{y \in X_{N-j}} V(x, y) .
$$

To understand the asymptotic behavior of $\varrho_{j}^{N}$ (for fixed $j$, when $N \rightarrow \infty$ ) we find convenient to rewrite the expression (2.13) in the following form:

$$
\begin{aligned}
\varrho_{j}^{N}\left(X_{j}\right)= & \frac{Z(N-j)}{Z(N)} e^{-\frac{\beta}{N} U\left(X_{j}\right)} \prod_{i=1}^{j} \int \mu^{N-j}\left(d X_{N-j}\right) \\
& e^{-\frac{\beta}{N} \sum_{N-j}^{\Sigma} \sum_{j}^{1} V\left(x_{i}, x_{k}\right)} e^{+\frac{j \beta}{N(N-j)} U\left(X_{N-j}\right)} .
\end{aligned}
$$

The limit we are considering is of mean field type. If the empirical distribution of the vortex system

$$
\frac{1}{N} \sum_{j=1}^{N} \delta_{x_{j}}(d x)
$$

[here $\delta_{y}(d x)$ denotes the Dirac measure concentrated on the point $y$ ] is going to converge, with large probability (weakly) to a (smooth) vorticity profile $\varrho$, then $\varrho_{j}^{N}$ are going to factorize:

$$
\varrho_{j}^{N} \rightarrow \varrho^{\otimes j} \quad \text { (weakly) }
$$

From (2.15), $\varrho$ must satisfy the following equation:

$$
\varrho(x)=Z^{-1} e^{-\beta V_{e}} e^{\frac{1}{2} \beta\left(\varrho, V_{Q}\right)},
$$

where

$$
\begin{gathered}
V_{\varrho}(x)=\int V(x, y) \varrho(y), \\
Z=\lim _{N \rightarrow \infty} \frac{Z(N)}{Z(N-1)}
\end{gathered}
$$

and $(\cdot, \cdot)$ denotes the usual scalar product.

The following identity is obvious:

$$
z e^{\frac{1}{2}\left\{\beta\left(\varrho, V_{\varrho}\right)\right.}=\int_{\Lambda} e^{-\beta V_{\varrho}} d x .
$$

In general, however, correlations can persist in the limit so that

$$
\varrho_{j}^{N} \rightarrow \int v(d \varrho) \varrho^{\otimes j} \quad \text { (weakly), }
$$

where $v$ is a measure on the space of probability densities. In this case, which cannot be excluded unless (2.18) possesses a unique solution, $v$ is expected to be concentrated on solutions of (2.18).

The above heuristic arguments can be made rigorous.

Theorem 2.1. Let $\left\{d \varrho_{j}\right\}_{j=1}^{\infty}$ be a weak cluster point (in the sense of the weak convergence of measures) of the sequence $\varrho_{j}^{N}$, i.e. there exists a subsequence $N_{k}$ for which

$$
\int d \varrho_{j}\left(X_{j}\right) \varphi\left(X_{j}\right)=\lim _{k \rightarrow \infty} \int d X_{j} \varrho_{j}^{N_{k}}\left(X_{j}\right) \varphi\left(X_{j}\right)
$$

for all $j$ and for all bounded and continuous $\varphi$. 
Then, the measures $d \varrho_{j}$ are absolutely continuous

$$
d \varrho_{j}\left(X_{j}\right)=\varrho_{j}\left(X_{j}\right) d X_{j}
$$

and the following representation holds:

$$
\varrho_{j}\left(x_{1} \ldots x_{j}\right)=\int v(d \varrho) \prod_{k=1}^{j} \varrho\left(x_{k}\right),
$$

where $v$ is a Borel probability measure on $L_{1}(\Lambda)$ endowed with the weak topology.

Furthermore, $v$ is concentrated on those solutions $\varrho \in L_{\infty}(\Lambda)$ of $(2.18)$ minimizing or maximizing, for $\beta>0$ or $\beta<0$ respectively, the free energy functional

$$
f(\varrho)=\frac{1}{\beta} \int_{\Lambda} \varrho \log \varrho d x+\frac{1}{2}(\varrho, V \varrho)
$$

with the constraints $\varrho \geqq 0, \int_{\Lambda} \varrho d x=1$.

Remarks. For $\beta>0$, we know that there exists a unique solution $\varrho$ to $(2.18)$ (obviously minimizing $f$ ) so that $\varrho_{j}^{N}$ converges (not only for subsequences) to $\varrho^{\otimes j}$. This factorization property is usually called "propagation of chaos."

For $-8 \pi<\beta<0$, uniqueness is not known for general domains (see the discussion in Sect. 7). However, for the ball we can also prove uniqueness. In this case, by means of Theorem 2.1, we obtain the same conclusions as for the positive temperature case namely the convergence of $\varrho_{j}^{N}$ to a product state.

For the stream function $\psi$, defined as

$$
-\Delta \psi=\varrho,
$$

as a consequence of (2.18) we obtain (1.1). We remark that the velocity field $u=\left(-\partial_{2} \psi, \partial_{1} \psi\right)$ satisfies the stationary two-dimensional Euler equation.

We shall prove Theorem 2.1 in Sect. 4. We shall follow the strategy of Messer and Spohn [31] who obtained the same result for a bounded, smooth interaction potential $V$. Here, we need extra bounds on the correlation functions which will be derived in Sect. 3.

\section{Estimates on the Correlation Functions}

In this section we prove an estimate on the correlation functions $\varrho_{j}^{N}$ which will allow us to prove Theorem 2.1.

Theorem 3.1. For $\beta \in(-8 \pi,+\infty)$, there exists a constant $C$ (depending only on $\beta$ and 1 ) such that

$$
\varrho_{j}^{N}\left(X_{j}\right) \leqq C^{j} e^{-\frac{\beta}{N} U\left(X_{\imath}\right)} \quad \text { on } \Lambda^{j} .
$$

Proof. Assume first $\beta>0$. By definition

$$
\varrho_{j}^{N}\left(X_{j}\right)=\frac{e^{-\frac{\beta}{N} U\left(X_{\jmath}\right)}}{Z(N)} \int d Y_{N-j} e^{-\frac{\beta}{N} W\left(X_{\jmath} \mid Y_{N-\jmath}\right)} e^{-\frac{\beta}{N} U\left(Y_{N-\jmath}\right)},
$$

where

$$
W(X \mid Y)=\sum_{\substack{x \in X \\ y \in Y}} V(x, y)
$$


Since $V$ is positive, we have

$$
\varrho_{j}^{N}\left(X_{j}\right) \leqq e^{-\frac{\beta}{N} U\left(X_{j}\right)} \frac{\Theta_{N}(N-j)}{Z(N)},
$$

where we set, for $k<N$ :

$$
\Theta_{N}(k)=\int d X_{k} e^{-\frac{\beta}{N} U\left(X_{k}\right)}
$$

We have thus in particular

$$
\Theta_{N}(k) \leqq \frac{\int d X_{k} e^{-\frac{\beta}{N} U\left(X_{k}\right)} \int d x_{k+1} e^{-\frac{\beta}{N} W\left(X_{k} \mid x_{k+1}\right)} e^{-\frac{\beta}{N} \gamma\left(x_{k+1}\right)}}{\inf _{X_{k}} \int d x_{k+1} e^{-\frac{\beta}{N} \gamma\left(x_{k+1}\right)-\frac{\beta}{N} W\left(X_{k} \mid x_{k+1}\right)}}-
$$

The denominator of the right-hand side of (3.6) can be estimated from below by:

$$
|\Lambda| \mathrm{e}^{-\frac{\beta}{N} m} e^{-\beta \frac{k}{N} \bar{V}}
$$

upon using the Jensen inequality and setting

$$
\begin{gathered}
m=\max _{y} \gamma(y), \\
\bar{V}=\frac{1}{|\Lambda|} \sup _{\Lambda} \int_{\Lambda} d y V(x, y) .
\end{gathered}
$$

In conclusion, we obtain

$$
\frac{\Theta_{N}(k)}{\Theta_{N}(k+1)} \leqq|\Lambda|^{-1} e^{\beta\left(\tilde{V}+\frac{m}{N}\right)}
$$

and estimate (3.1) follows with $C$ given by the right-hand side of (3.9).

For negative $\beta$ the estimate is a little more involved. To estimate the integral

$$
I=\int d Y_{N-j} e^{-\frac{\beta}{N} W\left(X_{\jmath} \mid Y_{N-j}\right)} e^{-\frac{\beta}{N} U\left(Y_{N-\jmath}\right)}
$$

we use the Hölder inequality with $p=\frac{N}{2 j}$ and, consequently $q=\frac{N}{N-2 j}$. We have, for a suitable constant $C_{1}$,

$$
\begin{aligned}
\int d Y_{N-j} e^{-\frac{\beta p}{N} W\left(X_{j} \mid Y_{N}-j\right)} & \leqq C_{1}^{-\frac{\beta p}{N} j(N-j)} \int d y_{1} \ldots d y_{N-j} \prod_{i=1}^{j} \prod_{k=1}^{N-j}\left(\frac{1}{\left|x_{i}-y_{k}\right|}\right)^{\frac{|\beta| p}{2 \pi N}} \\
& \leqq C_{1}^{-\frac{\beta p}{N} j(N-j)}\left(\prod_{i=1}^{j}\left\{\int d y\left(\frac{1}{\left|x_{i}-y\right|}\right)^{\frac{|\beta| p_{j}}{2 \pi N}}\right\}^{\frac{N-j}{j}}\right)
\end{aligned}
$$

Here we have applied once more Hölder's inequality. Since $\delta \equiv \frac{|\beta| p j}{2 \pi N}=\frac{|\beta|}{4 \pi}<2$,

$$
H \equiv \sup _{x} \int_{\Lambda} d y\left(\frac{1}{|x-y|}\right)^{\delta}<+\infty .
$$

Therefore, the right-hand side of (3.11) is bounded by

$$
C_{1}^{-\frac{\beta}{2}(N-j)} H^{(N-j)} \text {. }
$$


Thus, we only have to estimate

$$
\int d Y_{N-j} e^{-\frac{\beta}{N} \frac{N}{N-2 j} U\left(Y_{N-j}\right)} .
$$

The above expression can be written as

$$
\int d Y_{N-j} e^{-\frac{\beta}{N} \frac{1}{u} U\left(Y_{N-\jmath}\right)} e^{-\frac{\beta}{N}\left(\frac{N}{N-2 j}-\frac{1}{u}\right) U\left(Y_{N-\jmath}\right)}
$$

for $u>1$. Applying, once again, Hölder's inequality with conjugate exponent $u$ and $w$, we bound (3.15) by

$$
\begin{aligned}
& \Theta_{N}(N-j)^{\frac{1}{u}}\left(\int d Y_{N-j} e^{\left.-\frac{\beta}{N}\left(1+\frac{2 w j}{N-2 j}\right) U\left(Y_{N-J}\right)\right)^{\frac{1}{\omega}}}\right. \\
& =\Theta_{N}(N-j)^{1-\frac{j}{(N-2 j) \gamma}}\left(\int d Y_{N-1} e^{-(1+\gamma) \frac{\beta}{N} U\left(Y_{N-1}\right)}\right)^{\frac{j}{N-2 j}}
\end{aligned}
$$

The last equality follows by choosing $w=j^{-1}(N-2 j) \gamma$, where

$$
0 \leqq \gamma \leqq(8 \pi+\beta) / \beta
$$

(here $N$ is assumed to be large enough). By Lemma 2.1 the expression in parenthesis is bounded by $C_{2}^{N}$ so that, collecting all the above estimates, we obtain

$$
I \leqq C_{3}^{j} \Theta_{N}(N-j) \text {. }
$$

Finally, by the positivity of $V$ :

$$
\begin{aligned}
\Theta_{N}(N-j) & \leqq \frac{\int d Y_{N-j} \int d X_{j} e^{-\frac{\beta}{N} U\left(Y_{N}-\jmath\right)} e^{-\frac{\beta}{N} U\left(X_{\jmath}\right)} e^{-\frac{\beta}{N} W\left(X_{j} \mid Y_{N-\jmath}\right)}}{\int d X_{j} e^{-\frac{\beta}{N_{i} \Sigma_{1}^{j} \gamma\left(x_{\imath}\right)}}} \\
& \leqq C_{4}^{j} Z(N) .
\end{aligned}
$$

By (3.18), (3.17), (3.10), and (3.2) we obtain (3.1). This completes the proof.

\section{Proof of Theorem 2.1}

We denote by $\left\{\varrho_{j}\left(d X_{j}\right)\right\}_{j=1}^{\infty}$ the correlation measures obtained as weak cluster points of the sequence of measures $\left\{\varrho_{j}^{N}\left(x_{j}\right) d X_{j}\right\}_{j=1}^{N}$.

By the Hewitt-Savage theorem, there exists a probability Borel measure $v(d \varrho)$ on the space $\mathscr{M}_{1}^{+}$of all probability measures in $\Lambda$, endowed with the weak topology, for which

$$
\int \varrho_{j}\left(d X_{j}\right) d X_{j} f\left(X_{j}\right)=\int v(d \varrho) \int d \varrho\left(x_{1}\right) \ldots d \varrho\left(x_{j}\right) f\left(x_{1} \ldots x_{j}\right)
$$

for all $j$ and all bounded measurable functions $f: \Lambda^{j} \rightarrow \mathbb{R}$.

By Theorem 3.1, $\varrho_{j}\left(d X_{j}\right)$ is absolutely continuous. Denoting by $\varrho_{j}$ the densities we have:

$$
\left\|\varrho_{j}\right\|_{\infty} \leqq C^{j}
$$

Moreover, denoting by

$$
\varrho(f)=\int_{\Lambda} f(x) \varrho(d x) \quad d \varrho \in \mathscr{M}_{1}^{+}
$$


we have, for a positive $f$ and $p>1$,

$$
\begin{aligned}
\int v(d \varrho) \varrho(f)^{p} & =\int \varrho\left(x_{1} \ldots x_{p}\right) f\left(x_{1}\right) \ldots f\left(x_{p}\right) d x_{1} \ldots d x_{p} \\
& \leqq C^{p}\|f\|_{1}^{p} .
\end{aligned}
$$

Therefore:

$$
\|\varrho(f)\|_{L^{\infty}(v)} \leqq C\|f\|_{1}
$$

yielding:

$$
\varrho(d x)=\varrho(x) d x \quad \varrho \in L_{\infty}(\Lambda) \quad v \text { a.a. } \varrho .
$$

Since $v$ is supported on $L_{\infty}$ functions, the representation formula (4.1) can be written as

$$
\int d v(\varrho) \prod_{i=1}^{j} \varrho\left(x_{i}\right)=\varrho_{j}\left(x_{1} \ldots x_{j}\right) .
$$

For any pair $v$ and $\left\{\varrho_{j}\right\}_{j=1}^{\infty}$ satisfying (4.6) we define the free energy functional

$$
f(v)=\frac{1}{\beta} s(v)+\varepsilon(v)
$$

where

$$
\begin{aligned}
& s(v)=\lim _{j \rightarrow \infty} \frac{1}{j} \int d X_{j} \varrho_{j} \log \varrho_{j} \quad \text { (mean entropy), } \\
& e(v)=\frac{1}{2} \int d x d y \varrho_{2}(x, y) V(x, y) \quad \text { (mean energy). }
\end{aligned}
$$

By the subadditivity of the entropy, the limit (4.8) either exists or it is infinity. Moreover, it is well known that (see for instance [38])

$$
s(v)=\int v(d \varrho) s(\varrho),
$$

where

$$
s(\varrho)=\int \varrho \log \varrho d x .
$$

Note that, in physics, this entropy is usually $-s$.

We consider also the free energy functional:

$$
\begin{aligned}
F^{N} & =\frac{1}{\beta N} \int \mu^{N} \log \mu^{N}+\frac{1}{N^{2}} \int \mu^{N} U \\
& =-\frac{1}{\beta N} \log Z(N) .
\end{aligned}
$$

We want to prove that

$$
\lim F^{N}=f(v),
$$

where the above limit is taken on the subsequence for which $\varrho_{j}$ is the weak limit of $\varrho_{j}^{N}$ and $v$ is given by (4.6). In fact, by the convexity and subadditivity property of the entropy:

$$
\begin{aligned}
\frac{1}{j} \int \varrho_{j} \log \varrho_{j} & \leqq \liminf _{N \rightarrow \infty} \frac{1}{j} \int \varrho_{j}^{N} \log \varrho_{j}^{N} \\
& \leqq \liminf _{N \rightarrow \infty} \frac{1}{N} \int \mu^{N} \log \mu^{N}
\end{aligned}
$$


and thus

$$
s(v) \leqq \liminf \frac{1}{N} \int \mu^{N} \log \mu^{N}
$$

Moreover, it is easy to get

$$
\lim \frac{1}{N^{2}} \int \mu^{N}\left(x_{N}\right) U\left(x_{N}\right) d X_{N}=e(v)
$$

as a consequence of Theorem 3.1. Thus, we deduce

$$
\begin{aligned}
\liminf F^{N} \geqq f(v) & \text { for } \quad \beta>0, \\
\lim \sup F^{N} \leqq f(v) & \text { for } \quad \beta<0 .
\end{aligned}
$$

On the other hand $\mu^{N}$ minimizes for $\beta>0$ (maximizes for $\beta<0$ ) the free energy so that

$$
F^{N} \leqq \frac{1}{\beta^{N}} \int \varrho_{N} \log \varrho_{N}+\frac{1}{N^{2}} \int \varrho_{N} U_{N} \quad \text { if } \quad \beta>0
$$

and the reverse inequality holds for $\beta<0$. Thus, by (4.17) and (4.18) we finally obtain (4.13).

We finally prove that

$$
\begin{array}{rlrl}
f(v) & =\min _{\tilde{v} \in \mathcal{M}_{1}^{+}} f(\tilde{v}) & \beta>0 \\
& =\max _{\tilde{v} \in \mathscr{M}_{1}^{+}} f(\tilde{v}) & & \beta<0 .
\end{array}
$$

Consider $\beta>0$. Then:

$$
F^{N} \leqq \frac{1}{\beta N} \int \tilde{\varrho}_{N} \log \tilde{\varrho}_{N}+\frac{1}{N^{2}} \int \tilde{\varrho}_{N} U\left(X_{N}\right),
$$

where $\tilde{\varrho}_{j}$ and $\tilde{v}$ are related by (4.6). Thus we find

$$
f(v) \leqq f(\tilde{v}),
$$

and the reverse inequality holds for $\beta<0$.

In fact, the proof above together with the remark following Theorem 2.1 yields the following.

Corollary 4.1. If $\beta>0$ or if $\beta<0$ and we assume that there exists a unique $\varrho \in L_{\infty}(\Lambda)$ which maximizes $f(\varrho)$ over all $\tilde{\varrho} \in L_{1}(\Lambda), \int_{\Lambda} \varrho d x=1, \tilde{\varrho} \geqq 0$, then $\varrho_{j}^{N}$ and $\varrho_{j}^{N} \log \varrho_{j}^{N}$ converge a.e. and in $L_{1}\left(\Lambda^{j}\right)$ respectively to $\varrho_{j}=\prod_{i=1}^{j} \varrho\left(x_{i}\right)$ and $\varrho_{j} \log \varrho_{j}$.

Indeed, we then have $\varrho_{j}=\prod_{i=1}^{j} \varrho\left(x_{i}\right)$ and the above proof yields the convergence of $\int_{\Lambda^{j}} \varrho_{j}^{N} \log \varrho_{j}^{N}$ to $\int_{\Lambda^{j}} \varrho_{j} \log \varrho_{j^{\prime}}$. The strict convexity of the entropy allows to conclude.

Another proof can be made using (2.15) and some heuristic arguments which can be made rigorous as in the proof of Theorem 2.1. This yields the pointwise convergence while the $L_{1}$ convergence follows from the dominated convergence theorem and Theorem 3.1. 


\section{Solutions of the Mean Field Equation with Radial Symmetry}

If we consider the case when

$$
\Lambda=\left\{x \in \mathbb{R}^{2} /|x|<1\right\} \text { or } \Lambda=\left\{x \in \mathbb{R}^{2} / a<|x|<1\right\},
$$

where $a \in(0,1)$, that is the case of a connected domain with rotational symmetry (up to a simple dilation), we may look for radial solutions of Eq. (1.1). In addition, we know from the general results of Gidas, Ni, and Nirenberg [21], that if $\Lambda=\left\{x \in \mathbb{R}^{2} /|x|<1\right\}$ they are the only solutions such that, say, $\varrho \in L_{\infty}(\Omega)$ [or in fact $\left.\varrho \log \varrho \in L_{1}(\Lambda)\right]$. Such radial solutions that we denote by $\psi(r)$ satisfy

$$
\frac{1}{r} \frac{d}{d r}\left(r \frac{d}{d r} \psi\right)=-\frac{1}{\widehat{Z}} e^{-\beta \psi},
$$

which after using the classical change of variable $t=\log r$ becomes

$$
\frac{d^{2}}{d t^{2}} H=\beta \tilde{Z}^{-1} e^{H}
$$

where we set

$$
H(t)=-\beta \psi\left(e^{t}\right)+2 t
$$

Equation (5.2) corresponds to an autonomous, one dimensional Hamiltonian system which can be solved explicitly. Setting $y=e^{H}$, we deduce

$$
y(t)=\frac{4 E A \tilde{Z}}{\beta} e^{\sqrt{2 E t}}\left(1-A e^{\sqrt{2 E} t}\right)^{-2},
$$

where

$$
E=\frac{1}{2} \dot{H}^{2}-\frac{\beta}{\widetilde{Z}} e^{H}
$$

is the constant energy and $A$ is a constant to be determined by the normalization and boundary conditions. In the case when $\Lambda$ is the unit disk (that we denote by $\Lambda_{0}$ ) we have $\psi(1)=0$ and $\psi^{\prime}(1)$ can be determined by integrating the equation over $\Lambda_{0}$. We then find a solution for $\beta>-8 \pi$ determined by the choices

$$
A=\frac{\beta}{8 \pi+\beta}, \quad \tilde{Z}=\pi(1-A), \quad E=2 .
$$

The vorticity distribution $\varrho$ takes the form

$$
\varrho=-\Delta \psi=e^{-\beta \psi} \tilde{Z}^{-1}=\frac{1-A}{\pi} \frac{1}{\left(1-A r^{2}\right)^{2}} .
$$

We therefore observe that there are solutions of the mean field equation (1.1) if and only if $\beta>-8 \pi$ and that when $\beta \rightarrow-8 \pi$ this solution concentrates at 0 : indeed, $\varrho \rightarrow \delta_{0}$ weakly in the sense of measures.

In the case when $\Lambda=\Lambda_{a}=\left\{x \in \mathbb{R}^{2} / a<|x|<1\right\}$ [and $\left.a \in(0,1)\right]$, we obtain as before

$$
y(t)=\frac{4 E \tilde{Z} A}{\beta} e^{\sqrt{2 E} t}\left(1-A e^{\sqrt{2 E} t}\right)^{2},
$$


and we have to determine $E, A, \tilde{Z}$ by means of the normalization and the boundary conditions: $\psi(a)=\psi(1)=0$. In this way, we obtain for all $\beta \in \mathbb{R}$ a unique radial solution. Notice that in an annulus there is not a particular point at which radial solutions can concentrate as $\beta \rightarrow-8 \pi$.

Let us also notice that for such an annulus (or for similar geometries with one or many "holes"), one may impose different boundary conditions like $\psi(1)=0$, $\psi(a)=\alpha$. The analysis performed in the preceding sections easily adapts to that situation yielding similar results. Now, for a given $\alpha$ (which corresponds physically to a circulation assigned a priori), one finds in a similar way a unique radial solution for all $\beta \in \mathbb{R}$. We finally observe that in order to recover the solution in the disk from the solution in the annulus in the limit $a \rightarrow 0$, we need to prescribe a large value of $\alpha$ when $\beta$ is close to $-8 \pi$.

\section{A Mean Field Equation in $\mathbb{R}^{2}$}

Consider a system of $N$ vortices, with intensity $\alpha$, in all $\mathbb{R}^{2}$. The equation of motion is

$$
\dot{\chi}_{i}=-\frac{1}{2 \pi} \sum_{\substack{j=1 \\ j \neq i}}^{N} \nabla_{\chi_{i}}^{\perp} \log \left|x_{i}-x_{j}\right|, \quad i=1 \ldots N,
$$

where $\nabla^{\perp}=\left(\partial_{2},-\partial_{1}\right)$. It is well known that (6.1) admits the following first integrals:

$$
\begin{aligned}
& H=\frac{\alpha^{2}}{4 \pi} \sum_{\substack{i, j=1 \\
i \neq j}}^{N} \log \left|x_{i}-x_{j}\right| \quad \text { (energy), } \\
& M=\alpha \sum_{i=1}^{N} x_{i} \quad \text { (center of vorticity), } \\
& I=\alpha \sum_{i=1}^{N} x_{i}^{2} \quad \text { (moment of inertia) }
\end{aligned}
$$

connected with the time, translation and rotation invariance of the Hamiltonian (6.2) respectively. Therefore, the following measure

$$
\mu^{N}\left(d x_{1} \ldots d x_{N}\right)=\frac{1}{Z(N)} e^{-\tilde{\beta} H-\tilde{\lambda} I-\eta \cdot M}
$$

is invariant with respect to the evolution given by (6.1), for $\tilde{\lambda}>0, \widetilde{\beta} \in \mathbb{R}, \eta \in \mathbb{R}^{2}$. For positive $\tilde{\lambda}, I$ plays the role of an external field confining the system around the origin. Thus, all the considerations of Sects. 2 and 3 can be extended to this case with minor modifications. In particular the Mean Field equation takes the form (in the scaling $\alpha=\frac{1}{N}, \widetilde{\beta}=\beta N, \tilde{\lambda}=\lambda N$ )

$$
-\Delta \psi=\frac{1}{\widetilde{Z}} e^{-\beta \psi-\lambda|x|^{2}}
$$

associated, as above to the variational principles relative to the functionals

$$
f(\varrho)=\frac{1}{\beta} \int \varrho \log \varrho+\frac{1}{2} \int \varrho V_{\varrho}+\frac{\lambda}{\beta} \varrho|x|^{2} .
$$


Here we have eliminated the linear term in $\eta$ by means of a suitable change of coordinates.

Following the previous analysis we can prove the following theorem.

Theorem 6.1. Let $\beta \in(-8 \pi, \infty)$ and let $\lambda>0$. Then, there is a unique $\varrho \in L_{\infty}\left(\mathbb{R}^{2}\right)$ which maximizes for $\beta<0$ and minimizes for $\beta>0$ the functional $f$ given by (6.7). And there is a solution - determined up to a constant - of Eq. (6.6) with $\varrho=\frac{1}{\tilde{Z}} e^{-\beta \psi-\lambda|x|^{2}}$ such that $\int_{\mathbb{R}^{2}}|\nabla \psi|^{2}<\infty$. Moreover, $\varrho$ and $\psi$ are radially symmetric. Finally, the correlation functions $\varrho_{j}^{N}$ associated to the sequence of measures (6.5) converge pointwise and in $L_{1}$, as $N \rightarrow \infty$, to $\prod_{i=1}^{j} \varrho\left(x_{i}\right)$.

The arguments of Sect. 3 and 4 apply with minor modifications so that we have the convergence of the correlation functions and the existence of a limiting solution. We now apply the methods of Sect. 5 to find radial symmetric solutions for $\beta \in(-8 \pi,+\infty)$. Adding if necessary a constant to $\psi$, we may assume that $\tilde{Z}=1$ and we have to find a unique radial $\psi$ solving (6.6). We then set $H(t)=-\beta \psi(r)$ $-\lambda e^{2 t}+2 t$ with $t=\log r$ and we obtain

$$
\ddot{H}=\beta e^{H}-4 \lambda e^{2 t} .
$$

Unfortunately, since this system is not autonomous, we cannot give an explicit solution of (6.8). However, we can prove that there is only one trajectory for which $\widetilde{Z}=1$ and

$$
\lim _{t \rightarrow-\infty} \dot{H}(t)=2 .
$$

This condition follows from the fact that

$$
\lim _{r \rightarrow 0} r \psi^{\prime}(r)=0
$$

due to the regularity of $\psi$ (recall that $\varrho \in L_{\infty}$ ).

More precisely, we are interested in solutions of (6.8) behaving like $2 t+\chi$ as $t$ goes to $-\infty$. We remark that such solutions depend monotonically on $\chi$. Indeed by a change of the time scale: $2 t \rightarrow 2 t-\chi$, we see that increasing $\chi$, we decrease the force opposing the motion. Since

$$
\tilde{Z}=2 \pi \int_{-\infty}^{+\infty} e^{H} d t
$$

we also realize that $\tilde{Z}$ is increasing with $H$, so that to find $\chi$ such that the corresponding $\hat{Z}=1$, it is enough to observe that $\widetilde{Z} \rightarrow 0$ as $\psi \rightarrow-\infty$ and $\widetilde{Z} \rightarrow C$ as $\chi \rightarrow+\infty$ and $C>1$ if $\beta>-8 \pi$. The last statement follows by a direct computation. We conclude the proof by showing the uniqueness of maximizing solutions. This follows by a standard argument based on Schwarz symmetrization: given a nonsymmetric maximizer solution $\psi$, its symmetric rearrangement leaves $\widetilde{Z}$ invariant while the energy decreases. Thus, a maximizing solution must be symmetric.

It remains to investigate the problem of the behavior of the solutions when $\beta \rightarrow 8 \pi_{+}$. As in the case of the disk the solutions converge weakly in the sense of measures to the Dirac measure supported at the origin. We shall give later on the proof of this statement. 


\section{Some Properties of Solutions of the Mean Field Equation}

We consider in this section solutions $\psi \in\left(H_{0}^{1}(\Lambda) \cap L_{\infty}(\Lambda)\right)$ of the mean field equation

$$
-\Delta \psi=e^{-\beta \psi}\left(\int_{\Lambda} e^{-\beta \psi}\right)^{-1} \text { in } \Lambda, \psi=0 \text { on } \partial \Lambda .
$$

We will only consider the case when $\beta<0$ in this section since the case $\beta>0$ is completely solved in [22]. Let us first remark that $\psi$ is in fact smooth [ $C^{\infty}(\bar{\Lambda})$ if $\Lambda$ is smooth]: this follows easily from elliptic regularity. Let us also recall that if $\varrho \in L_{1}(\Lambda), \varrho \geqq 0$, and $\int_{\Lambda} \varrho=1$ and if $\psi$ solves

$$
-\Delta \psi=\varrho \text { in } \Lambda, \quad \psi=0 \text { on } \partial \Lambda
$$

then $\psi$ and $\nabla \psi$ are relatively compact in $L_{p}(\Lambda)$ for all $p<\infty, p<2$ respectively. Furthermore, if $\varrho \log \varrho \in L_{1}(\Lambda)$, then $\psi \in H_{0}^{1}(\Lambda) \cap C^{0}(\bar{\Lambda})$ (and is thus smooth). Also, if $\psi \in H_{0}^{1}(\Lambda)$ solves (7.1) then (see [31]) $e^{\mu \psi^{2}} \in L_{1}(\Lambda)$ for all $\mu<\infty$, therefore in particular $\varrho \in L_{q}(\Lambda)$ for all $q<\infty$ and $\psi \in C^{0}(\bar{\Lambda})$.

We begin this section with a few observations on general solutions of (7.1). First of all, if $\Lambda$ is starshaped, Pohozaev's identity ([34]) yields

$$
\frac{2}{(-\beta)}\left(\int_{\Lambda} e^{-\beta \psi}\right)^{-1}\left(\int_{\Lambda} e^{-\beta \psi}-1\right)=\frac{1}{2} \int_{\partial \Lambda}(x \cdot v)\left|\frac{\partial \psi}{\partial v}\right|^{2} d \sigma
$$

where $v$ is the unit outer normal to $\partial \Lambda$, and we assume that $\Lambda$ is starshaped with respect to 0 (translating the origin if necessary). Let us recall that (7.3) follows from the multiplication of (7.1) by $x \cdot \nabla \psi$ and integrations by parts. We will say that $\Lambda$ is strictly starshaped if there exists a constant $\alpha_{0}>0$ such that

$$
(x \cdot v)\left(\int_{\partial \Lambda} d \sigma\right)^{-1} \geqq \alpha_{0} \quad \text { on } \partial \Lambda .
$$

(Observe that if $\Lambda$ is a ball, we may take $\alpha_{0}=\frac{1}{2 \pi}$.) Then, (7.3) and (7.4) imply

$$
\frac{2}{|\beta|}>\frac{\alpha_{0}}{2}\left(\int_{V \Lambda}-\frac{\partial \psi}{\partial v} d \sigma\right)^{2}=\frac{\alpha_{0}}{2}
$$

since $\int_{\partial \Lambda}-\frac{\partial \psi}{\partial v} d \sigma=\int_{\Lambda}-\Delta \psi=1$. And we have shown the

Proposition 7.1. Let us assume that $\Lambda$ is strictly starshaped. Then, there does not exist a solution of (7.1) if $\beta \leqq-\frac{4}{\alpha_{0}}$.

We recover in particular the fact shown in Sect. 5 that if $\Lambda$ is a ball there does not exist a solution of (7.1) if $\beta \leqq-8 \pi$. On the other hand, for domains which are not starshaped, solutions may exist for all $\beta<0$ as we saw in Sect. 5 when $A$ is an annulus. It might be worth, at this stage, going back to the example of the annulus to show how radial solutions may be built for all $\beta<0$ by a variational argument.

Proposition 7.2. Let $\beta \in(-8 \pi, 0)$ and $\Lambda=\left\{x \in \mathbb{R}^{2} / a<|x|<1\right\}$, where $a \in(0,1)$. Then, there exists a unique radial solution $\psi$ of (7.1) which is the unique maximizer of

$$
\max \left\{-\frac{1}{\beta} \log \left(\int_{\Lambda} e^{-\beta \psi}\right)-\frac{1}{2} \int_{\Lambda}|\nabla \psi|^{2} / \psi \in H_{0}^{1}(\Lambda), \psi \text { is radial }\right\}
$$


and $\varrho=-\Delta \psi$ is the unique maximizer of

$$
\begin{aligned}
\max & \left\{\frac{1}{2} \int_{\Lambda \times \Lambda} \varrho(x) \varrho(y) V(x, y)+\frac{1}{\beta} \int_{\Lambda} \varrho \log \varrho / \varrho \in L_{1}(\Lambda), \varrho \geqq 0,\right. \\
& \left.\int_{\Lambda} \varrho=1, \varrho \text { is radial }\right\} .
\end{aligned}
$$

We only have to explain why (7.3) for example admits a maximum in view of the analysis of radial solutions performed in Sect. 5. This follows easily from the fact that, if $\psi \in H_{0}^{1}(\Lambda)$ is radial, we have for all $r \in(a, 1)$,

$$
\begin{aligned}
|\psi(r)| & =\left|\int_{r}^{1} \psi^{\prime} d s\right| \leqq\left(\int_{a}^{1}\left|\psi^{\prime}\right|^{2} s d s\right)^{1 / 2} a^{-1 / 2} \\
& =(2 \pi a)^{-1 / 2}\|\nabla \psi\|_{L_{2}} .
\end{aligned}
$$

By inequality (7.5) it follows that $-\frac{1}{\beta} \log \int_{\Lambda} e^{-\beta \psi}-\frac{1}{2} \int_{\Lambda}|\nabla \psi|^{2}$ is bounded in $H_{0}^{1}(\Lambda)$ and that the maximum is actually achieved since, for a maximizing sequence $\psi_{n}$, the bound $\left\|\nabla \psi_{n}\right\|_{L_{2}}<$ const holds.

It is possible to give other examples of domains with holes for which solutions exist for some $\beta<-8 \pi$ (domains with symmetries for instance): it might be possible to have some results à la Bahri-Coron [5].

Let us come back to the solutions in $\mathbb{R}^{2}$ discussed in the previous section. An application of the Pohozaev identity applied to the domain $\Lambda(R)=\{x /|x|<R\}$ yields:

$$
\begin{aligned}
-\frac{1}{2} R \oint_{\partial \Lambda(R)}\left(\frac{\partial \psi}{\partial n}\right)^{2} d \sigma= & -\frac{R e^{-\lambda R^{2}}}{\beta} \oint_{\partial \Lambda(R)} e^{-\beta \psi} d \sigma \cdot \frac{1}{\int_{\Lambda(R)} e^{-\beta \psi-\lambda x^{2}}} \\
& +\frac{2}{\beta}-\frac{2 \lambda}{\beta} \frac{\int_{\Lambda(R)} x^{2} e^{-\beta \psi-\lambda x^{2}}}{\int_{\Lambda(R)} e^{-\beta \psi-\lambda x^{2}}} .
\end{aligned}
$$

Taking the limit $R \rightarrow \infty$, we find the inequality $\beta>-8 \pi$. Moreover, for $\beta=-8 \pi$ we get $\int \varrho(x) x^{2} d x=0$ which ensures the concentration of the solution.

We now turn to the class of solutions we are really interested in namely those which satisfy a variational principle involving the functionals

$$
\bar{g}(\psi)=-\frac{1}{\beta} \log \left(f_{\Lambda} e^{-\beta \psi}\right)-\frac{1}{2} \int_{\Lambda}|\nabla \psi|^{2} \quad \text { on } H_{0}^{1}(\Lambda)
$$

or

$$
\bar{f}(\varrho)=\frac{1}{2} \int_{\Lambda \times \Lambda} \varrho(x) \varrho(y) V(x, y)+\frac{1}{\beta} \int_{\Lambda} \varrho \log \varrho+\frac{1}{\beta} \log |\Lambda| \quad \text { on } \mathscr{P} \text {, }
$$

where $\mathscr{P}=\left\{\varrho \in L_{1}(\Lambda), \varrho \geqq 0, \int_{\Lambda} \varrho=1\right\}, f=\frac{1}{|\Lambda|} \int_{\Lambda}$.

We first recall an inequality due to Moser [32] (see also [33, 26]): there exists a positive constant $C$ such that for all $\psi \in H_{0}^{1}(\Lambda)$,

$$
\frac{1}{8 \pi} \log \left(\int_{\Lambda} e^{8 \pi \psi}\right)-\frac{1}{2} f|\nabla \psi|^{2} \leqq C .
$$


To simplify notations, we will introduce $\bar{\beta}=-\beta$ and when needed we will recall the dependence of $g$ and $f$ upon $\bar{\beta}$ by writing $g_{\bar{\beta}}$ or $f_{\bar{\beta}}$.

The following result is essentially a simple consequence of (7.9).

Proposition 7.3.1) We have for all $\bar{\beta}>0, I_{\bar{\beta}}(\Lambda)=\sup \bar{g}_{\bar{\beta}}(\psi)<+\infty$ if and only if $\bar{\beta} \leqq 8 \pi$

$$
I_{\bar{\beta}}(\Lambda)=\sup _{\varrho \in \mathscr{P}} \bar{f}(\varrho) \text { for all } \bar{\beta}>0 \text {, }
$$

$I_{\bar{\beta}}(\Lambda)$ is a continuous increasing function of $\bar{\beta}$ on $[0,8 \pi]$,

$$
I_{\bar{\beta}}(\Lambda)=I_{\bar{\beta}}(t \Lambda) \leqq I_{\bar{\beta}}\left(\Lambda_{0}\right) \text { for all } \bar{\beta}>0, t>0,
$$

where $\Lambda_{0}=\left\{x \in \mathbb{R}^{2} /|x|<1\right\}$.

2) If $\bar{\beta} \in(0,8 \pi)$, every maximizing sequence of $I_{\bar{\beta}}(\Lambda)$ converges, up to the extraction of a subsequence, to a maximizer. In particular, there exists a maximizer $(\psi, \varrho)$ of $I_{\bar{\beta}}(\Lambda)$ if $\bar{\beta} \in(0,8 \pi)$.

3) There does not exist a maximizer of $I_{8 \pi}\left(\Lambda_{0}\right)$.

Remarks. Or course, the convergence in 2) depends on which (equivalent) formulation of $I_{\bar{\beta}}(\Lambda)$ we choose: if we take the maximization in $\psi$ as in (7.9), the convergence is in $H_{0}^{1}(\Lambda)$ while if we maximize in $\varrho$ as in (7.10) the convergence is the following:

$$
\begin{gathered}
\varrho_{n} \stackrel{n}{\longrightarrow} \varrho \text { in } L_{1}, \quad \varrho_{n} \log \varrho_{n}+1 \stackrel{n}{\longrightarrow} \varrho \log \varrho+1 \text { in } L_{1}, \\
\int V(x, y) \varrho_{n}(y) d y \stackrel{n}{\longrightarrow} \int V(x, y) \varrho(y) d y \text { in } H_{0}^{1} .
\end{gathered}
$$

Let us observe that, by convexity, $\varrho \log \varrho+1 \geqq \varrho \geqq 0$.

We now prove Proposition 7.3: the claim (7.11) follows easily from Jensen's inequality and (7.9) - the continuity is a straightforward exercise using the bounds on $\psi$ in $H_{0}^{1}(\Lambda)$. The claim (7.12) follows from scaling arguments and Schwarz symmetrization which decreases the Dirichlet integral. Or course, (7.9) shows that $I_{\bar{\beta}}(\Lambda) \leqq I_{8 \pi}(\Lambda) \leqq I_{8 \pi}\left(\Lambda_{0}\right)<\infty$ if $\bar{\beta} \leqq 8 \pi$. But it also shows that if $\bar{\beta}<8 \pi$, maximizing sequences of $\bar{I}_{\bar{\beta}}(\Lambda)$ are bounded say in $H_{0}^{1}(\Lambda)$, bounds which imply immediately part 2) of Proposition 7.3. Part 3) follows from our analysis in Sect. 5 since we showed there that (7.1) does not admit a solution if $\beta=-8 \pi$ and $\Lambda=\Lambda_{0}$. In fact, this also shows that $I_{\bar{\beta}}\left(\Lambda_{0}\right)=+\infty$ if $\bar{\beta}>8 \pi$ since otherwise we would find a maximum for $I_{8 \pi}\left(\Lambda_{0}\right)$.

Next, if $\bar{\beta}>8 \pi$ and if we take a small enough ball $\Lambda^{\prime}$ included in $\Lambda$ we observe that if $\psi \in H_{0}^{1}\left(\Lambda^{\prime}\right)$ then

$$
\bar{g}_{\Lambda^{\prime}}(\psi) \leqq \bar{g}_{\Lambda}(\psi)+\frac{1}{\bar{\beta}} \log \frac{|\Lambda|}{\left|\Lambda^{\prime}\right|}-\frac{1}{\beta} \log \left(1+\frac{\left|\Lambda-\Lambda^{\prime}\right|}{\int_{\Lambda^{\prime}} e^{-\beta \psi}}\right),
$$

where we recall the dependence of $\bar{g}$ upon $\Lambda$ by writing $\bar{g}_{\Lambda}$ and where we extend $\psi$ by 0 into a function in $H_{0}^{1}(\Lambda)$. And (7.17) shows

$$
I_{\bar{\beta}}\left(\Lambda^{\prime}\right) \leqq I_{\bar{\beta}}(\Lambda)+\frac{1}{\bar{\beta}} \log \frac{|\Lambda|}{\left|\Lambda^{\prime}\right|}
$$

and we conclude since $I_{\bar{\beta}}\left(\Lambda^{\prime}\right)=I_{\bar{\beta}}\left(\Lambda_{0}\right)=+\infty$ if $\bar{\beta}>8 \pi$. 
There only remains to show (7.10) which follows from the simple observation that, if $\psi$ solves (7.1) and $\varrho=-\Delta \psi$, then

$$
\bar{f}(\varrho)=\bar{g}(\psi) .
$$

We now want to investigate the maximization for $\bar{\beta}=8 \pi$. The main result in that direction is the following theorem. Let us mention before that, when we consider a maximizing sequence $\varrho_{n}$ for $I_{8 \pi}(\Lambda)$, we mean that $\varrho_{n}$ is a maximizing sequence for the problem defined in (7.10). Recall also that we denote by $\tilde{\gamma}(x, y)$ the regular part of the Green's function $V(x, y)$ and by $\gamma(x)=\frac{1}{2} \tilde{\gamma}(x, x)$ and that $\Lambda_{0}=\left\{x \in \mathbb{R}^{2} /|x|<1\right\}$.

Theorem 7.1. 1) The following inequality holds

$$
I_{8 \pi}(\Lambda) \geqq I_{8 \pi}\left(\Lambda_{0}\right)+\max _{x \in A} \gamma(x)+\frac{1}{8 \pi} \log \frac{\left|\Lambda_{0}\right|}{|\Lambda|} .
$$

In addition, every maximizing sequence $\varrho_{n}$ of $I_{8 \pi}(\Lambda)$ remains bounded that is $\int_{\Lambda} \varrho_{n} \log \varrho_{n}+1$ remains bounded if and only if we have

$$
I_{8 \pi}(\Lambda)>I_{8 \pi}\left(\Lambda_{0}\right)+\max _{x \in \Lambda} \gamma(x)+\frac{1}{8 \pi} \log \frac{\left|\Lambda_{0}\right|}{|\Lambda|} .
$$

In particular, if (7.21) holds, there exists a maximum of $I_{8 \pi}(\Lambda)$.

2) If $I_{8 \pi}(\Lambda)=I_{8 \pi}\left(\Lambda_{0}\right)+\max _{x \in \Lambda} \gamma(x)+\frac{1}{8 \pi} \log \frac{\left|\Lambda_{0}\right|}{|\Lambda|}$, there exists an unbounded maximizing sequence $\varrho_{n}$. And any such maximizing sequence, up to the extraction of a subsequence, satisfies the following properties: denoting by $\psi_{n}$ the solution of

$$
-\Delta \psi_{n}=\varrho_{n} \text { in } \Lambda, \quad \psi_{n} \in H_{0}^{1}(\Lambda),
$$

then $\varrho_{n},\left|\nabla \psi_{n}\right|^{2}\left\|\nabla \psi_{n}\right\|_{L_{2}}^{-1}, \varrho_{n} \log \varrho_{n}\left(\int_{\Lambda} \varrho_{n} \log \varrho_{n}\right)^{-1}$ converge weakly, in the sense of measures, to $\delta_{x_{0}}$ where $x_{0} \in \Lambda$ is a maximum point of $\gamma$. Furthermore, $\tilde{\varrho}_{n}=\varrho_{n}\left(x+x_{0}\right)$ (extended by 0 to $\left.D \Lambda_{0}\right)$ is a maximizing sequence of $I_{8 \pi}\left(D \Lambda_{0}\right)$, where $D>0$ is such that $\Lambda \subset x_{0}+D \Lambda_{0}$.

Remarks. 1. Of course, if $\Lambda=\Lambda_{0}, \max _{x \in \Lambda_{0}} \gamma(x)=\gamma(0)=0$ and since $I_{8 \pi}\left(\Lambda_{0}\right)$ is not achieved, we know that any maximizing sequence concentrates (in the above manner) at 0 .

2. We shall give in the sequel an example of a domain $\Lambda$ satisfying (7.21). However, we do not know an example of a domain $\Lambda$ for which the equality holds (except of course in the case when $\Lambda=\Lambda_{0}$ ).

3. A particular exemple of a maximizing sequence of $I_{8 \pi}(\Lambda)$ is obtained by maximizing $I_{\bar{\beta}}(\Lambda)$ for $\bar{\beta}<8 \pi$ and letting $\bar{\beta}$ go to $8 \pi$.

4. It is possible to precise the behavior of some maximizing sequences and since this analysis does not seem to help elucidating the alternative between maximization and concentration, we will not continue this analysis here.

5. The set of maxima of $\gamma$ may contain several points, even for simply connected domains. It may be a curve as in the case of the annulus. However it is known that if $\Lambda$ is convex and different from an infinite strip, $\gamma$ has only one critical point (see $[23,24])$. One could conjecture that the concentration does happen in this case. We shall show later on that this is not true. 
6. There is a physical reason why, if concentration occurs, it must be localized at a critical point of $\gamma$. Actually, $\gamma$ is the Hamiltonian for the motion of a single point vortex in $\Lambda$ so that the critical points of $\gamma$ are the equilibria of such a motion. Since a solution $\varrho$ of the mean field equation is the vorticity profile of a stationary solution of the Euler equation, whenever it degenerates in a $\delta$-function, its support "has" to be an equilibrium for the point vortex motion.

Proof of Theorem 7.1. The proof is rather long so that we split it in the following steps.

Step 1. Proof of (7.20) and the "only if part" of the claim 1).

Step 2. We take $\left(\varrho_{n}, \psi_{n}\right)$, the sequence of maxima of $I_{8 \pi-\frac{1}{n}}(\Lambda)$ as a maximizing sequence for $I_{8 \pi}(\Lambda)$ and prove that if it is unbounded, it must satisfy the behavior of claim 2). In this case (7.20) becomes an identity.

Step 3. The same claim as the previous step can be proved for an arbitrary unbounded sequence together with the proof of claim 2) and the "if" part of claim 1).

Proof of Step 1. Choose a maximum point $x_{0}$ of $\gamma$ and $\delta>0$ so that

$$
\left\{x /\left|x-x_{0}\right|<\delta\right\} \subset A \text {. }
$$

Then, consider the explicit maximizing sequence $\tilde{\varrho}_{n}$ for $I_{8 \pi}\left(\Lambda_{0}\right)$ built in Sect. 5 by solving $I_{8 \pi-\frac{1}{n}}\left(\Lambda_{0}\right)$ and set

$$
\varrho_{n}(x)=\frac{1}{\delta^{2}} \tilde{\varrho}_{n}\left(\frac{x-x_{0}}{\delta}\right) .
$$

One then easily checks that

$$
\begin{aligned}
\bar{f}_{\Lambda}\left(\varrho_{n}\right)= & \bar{f}_{\Lambda_{0}}\left(\tilde{\varrho}_{n}\right)+\frac{1}{8 \pi} \log \frac{\left|\Lambda_{0}\right|}{|\Lambda|}+\frac{1}{2} \iint_{\Lambda \times \Lambda} \varrho_{n}(x) \varrho_{n}(y) \tilde{\gamma}(x, y) \\
& -\frac{1}{2} \int_{\Lambda_{0} \times \Lambda_{0}} \tilde{\gamma}_{0}(x, y) \tilde{\varrho}_{n}(x) \tilde{\varrho}_{n}(y),
\end{aligned}
$$

where $\tilde{\gamma}_{0}$ is the regular part of the Green function in $\Lambda_{0}$.

Letting $n$ go to $+\infty$, we find

$$
I_{8 \pi}(\Lambda) \geqq I_{8 \pi}\left(\Lambda_{0}\right)+\frac{1}{8 \pi} \log \frac{\left|\Lambda_{0}\right|}{|\Lambda|}+\gamma\left(x_{0}\right),
$$

since $\varrho_{n} \stackrel{n}{\rightarrow} \delta_{x_{0}}$ and $\tilde{\gamma}_{0}(0,0)=0$. If the equality in (7.25) holds we have just constructed an unbounded maximizing sequence. This proves Step 1.

Proof of Step 2. Let $\left(\psi_{n}, \varrho_{n}\right)$ be maximizing solution of the mean field equation for $\beta_{n}=-8 \pi+\frac{1}{n}$. By virtue of Proposition 7.3, this is a maximizing sequence for $I_{8 \pi}(\Lambda)$. Suppose the sequence is unbounded and define the following probability measures:

$$
\begin{aligned}
& d \mu_{1}^{n}=\frac{\left|\nabla \psi_{n}\right|^{2}}{\left\|\nabla \psi_{n}\right\|_{L_{2}}^{2}}, \quad d \mu_{2}^{n}=\frac{\varrho_{n} \psi_{n} d x}{\left\|\nabla \psi_{n}\right\|_{L_{2}}^{2}} \\
& d \mu_{3}^{n}=\varrho_{n} d x, \quad d \mu_{4}^{n}=\frac{\varrho_{n} \log \varrho_{n}+1}{\int \varrho_{n} \log \varrho_{n}+1} .
\end{aligned}
$$


The above measures converge weakly (up to the extraction of subsequence), in the sense of measures, to some probability measures denoted by $\mu_{1}, \mu_{2}, \mu_{3}, \mu_{4}$ on $\bar{\Lambda}$. We now prove that $\mu_{1}=\mu_{2}=\mu_{3}=\mu_{4}=\delta_{x_{0}}, x_{0} \in \bar{\Lambda}$. find:

We first show that $\mu_{1}=\mu_{2}$. Indeed, multiplying $-\Delta \psi_{n}=\varrho_{n}$ by $\psi_{n} \varphi, \varphi \in C^{1}(\bar{\Lambda})$ we

$$
\int_{\Lambda}\left|\nabla \psi_{n}\right|^{2} \varphi+\int_{A} \nabla \psi_{n} \cdot \nabla \varphi \psi_{n}=\int \varrho_{n} \psi_{n} \varphi .
$$

Since $\int \varrho_{n}=1, \psi_{n}$ is bounded in $L_{p}$ for all $p<\infty$ and in particular in $L_{2}$. Dividing (7.27) by $\left\|\nabla \psi_{n}\right\|_{L_{2}}^{2}$ and letting $n \rightarrow \infty$ we obtain

$$
\int \varphi d \mu_{1}=\int \varphi d \mu_{2}
$$

provided that $\left\|\nabla \psi_{n}\right\|_{L_{2}} \rightarrow \infty$. This follows easily by the fact that the sequence is unbounded.

We now prove that $\mu_{1}=\frac{1}{2}\left(\mu_{3}+\mu_{4}\right)$. By the mean field equation and identity (7.27) we have:

$$
\begin{aligned}
\frac{1}{8 \pi-\frac{1}{n}} \int\left(\varrho_{n} \log \varrho_{n}+1\right) \varphi & =\int\left|\nabla \psi_{n}\right|^{2} \varphi+\int \nabla \psi_{n} \cdot \nabla \varphi \psi_{n} \\
& +\int \varphi-\frac{1}{\left(8 \pi-\frac{1}{n}\right)} \log \int e^{-\beta_{n} \psi_{n}} \int \varrho_{n} \varphi .
\end{aligned}
$$

On the other hand, by the variational principles, we have:

$$
\frac{\frac{1}{8 \pi} \log \int e^{-\beta_{n} \psi_{n}}}{\frac{1}{2}\left\|\nabla \psi_{n}\right\|_{L_{2}}^{2}} \rightarrow 1, \quad \frac{\frac{1}{8 \pi} \int\left(\varrho_{n} \log \varrho_{n}+1\right)}{\frac{1}{2}\left\|\nabla \psi_{n}\right\|_{L_{2}}^{2}} \rightarrow 1 .
$$

Thus, dividing by $\left\|\nabla \psi_{n}\right\|_{L_{2}}^{2}$ the identity (7.29), we obtain

$$
\int d \mu_{1} \varphi=\frac{1}{2}\left(\int d \mu_{3} \varphi+\int d \mu_{4} \varphi\right) .
$$

Finally, we want to prove that $\mu_{1}=\mu_{3}=\mu_{4}$ and that this measure reduces to a Dirac mass. Consider $\varphi \in C^{1}(\bar{\Lambda})$ and assume $\varphi \geqq 0, \int_{\Lambda} \varphi d \mu_{3}>0$. Then, for $n$ large enough, $\int \varphi \varrho_{n}>0$ therefore $\bar{f}\left(\varrho_{n} \varphi\left(\int_{\Lambda} \varrho \varphi\right)^{-1}\right) \leqq C_{0}=I_{8 \pi}(\Lambda)$, that is

$$
\begin{gathered}
\frac{1}{2} \iint_{\Lambda \times \Lambda} \varrho_{n}(x) \varphi(x) \varrho_{n}(y) \varphi(y) V(x, y) d x d y\left(\int_{\Lambda} \varrho_{n} \varphi\right)^{-2} \\
-\frac{1}{8 \pi} \int_{\Lambda} \varrho_{n} \varphi \log \frac{\varrho_{n} \varphi}{\int_{\Lambda} \varrho_{n} \varphi}\left(\int_{\Lambda} \varrho_{n} \varphi\right)^{-1} \leqq C_{0} .
\end{gathered}
$$

We then observe that

$$
\begin{aligned}
& \iint_{\Lambda \times \Lambda} \varrho_{n}(x) \varphi(x) \varrho_{n}(y) \varphi(y) V(x, y) \\
& \quad=\int_{\Lambda} \varrho_{n} \psi_{n} \varphi^{2}+\iint_{\Lambda \times \Lambda} \varrho_{n}(x) \varrho_{n}(y)[\varphi(y)-\varphi(x)] V(x, y),
\end{aligned}
$$


and since $\varphi \in C^{1}(\bar{\Lambda})$, the second term in the right-hand side is bounded. Therefore, dividing (7.35) by $\int_{\Lambda}\left|\nabla \psi_{n}\right|^{2}=\int_{\Lambda} \varrho_{n} \psi_{n}$ and using the above information, we deduce

$$
\int_{\Lambda} \varphi^{2} d \mu_{1}=\int_{\Lambda} \varphi^{2} d \mu_{2} \leqq\left(\int_{\Lambda} \varphi d \mu_{3}\right) \cdot\left(\int_{\Lambda} \varphi d \mu_{4}\right) .
$$

Replacing $\varphi$ by $\varphi+\delta$ with $\delta>0$ and letting $\delta$ go to 0 , we see that this inequality holds in fact for all $\varphi \geqq 0, \varphi \in C^{1}(\bar{\Lambda})$ and by density for all $\varphi \geqq 0, \varphi \in C^{0}(\bar{\Lambda})$. Since $\mu_{1}=\frac{\mu_{3}+\mu_{4}}{2}$, this implies in particular

$$
\int_{\Lambda} \varphi^{2} d \mu_{1} \leqq\left(\int_{\Lambda} \varphi d \mu_{1}\right)^{2}, \quad \text { for all } \varphi \geqq 0, \varphi \in C^{0}(\bar{\Lambda}),
$$

and it is well-known that this implies $\mu_{1}=\delta_{x_{0}}$ for some $x_{0} \in \bar{\Lambda}$. We then easily conclude that $\mu_{1}=\mu_{2}=\mu_{3}=\mu_{4}=\delta_{x_{0}}$.

Notice that we have only shown that the "concentration" point $x_{0}$ belongs to $\bar{\Lambda}$. We finally prove that $x_{0}$ maximizes $\gamma$ so that, in particular, $x_{0} \in \Lambda$. To this end we observe that

$$
\begin{aligned}
\bar{f}_{\Lambda}\left(\varrho_{n}\right)= & \bar{f}_{D \Lambda_{0}}\left(\varrho_{n}\right)+\frac{1}{8 \pi} \log \frac{\left|D \Lambda_{0}\right|}{|\Lambda|} \\
& +\frac{1}{2} \iint_{\Lambda \times \Lambda} \varrho_{n}(x) \varrho_{n}(y) \tilde{\gamma}(x, y) d x d y \\
& -\frac{1}{2} \int_{\left(x_{0}+D \Lambda_{0}\right) \times\left(x_{0}+D \Lambda_{0}\right)} \varrho_{n}(x) \varrho_{n}(y) \tilde{\gamma}_{0}\left(x-x_{0}, y-y_{0}\right) d x d y,
\end{aligned}
$$

where $\tilde{\gamma}_{0}$ is the regular part of the Green's function in $D \Lambda_{0}$. In particular,

$$
\begin{aligned}
I_{8 \pi}(\Lambda)-\delta_{n} \leqq & \bar{f}_{\Lambda}\left(\varrho_{n}\right) \leqq I_{8 \pi}\left(\Lambda_{0}\right)+\frac{1}{8 \pi} \log \frac{\left|\Lambda_{0}\right|}{|\Lambda|} \\
& +\frac{1}{2} \iint_{\Lambda \times \Lambda} \varrho_{n}(x) \varrho_{n}(y) \tilde{\gamma}(x, y) d x d y+\delta_{n},
\end{aligned}
$$

where $\delta_{n}>0, \delta_{n} \rightarrow 0$. Since $\varrho_{n} \underset{n}{\rightarrow} \delta_{x_{0}}$, this shows that $x_{0} \in \Lambda$ and we deduce from (7.36) letting $n$ go to $+\infty$,

$$
I_{8 \pi}(\Lambda) \leqq I_{8 \pi}\left(\Lambda_{0}\right)+\frac{1}{8 \pi} \log \frac{\left|\Lambda_{0}\right|}{|\Lambda|}+\gamma\left(x_{0}\right) .
$$

This inequality combined with (7.20) concludes the proof of Theorem 7.1.

Proof of Step 3. Consider an unbounded maximizing sequence $\left\{\varrho_{n}\right\}$. We want to modify it into another maximizing sequence close to the previous one which is now an approximate solution to the mean field equation.

Let $\psi_{n}$ be a solution of the Poisson equation

$$
-\Delta \psi_{n}=\varrho_{n} \text { in } \Lambda, \quad \psi_{n} \in H_{0}^{1}(\Lambda) .
$$

We argue as in Lions [29] using I. Ekeland's perturbed optimization principle [17] in the space $L_{1}(\Lambda) \cap H^{-1}(\Lambda)$, and we find a new maximizing sequence $\tilde{\varrho}_{n}$ such that $\varrho_{n}-\tilde{\varrho}_{n} \stackrel{n}{\longrightarrow} 0$ in $L_{1}(\Lambda) \cap H^{-1}(\Lambda)$ and

$$
\tilde{\psi}_{n}-\frac{1}{8 \pi} \log \tilde{\varrho}_{n}=\varepsilon_{n}+\theta_{n} \quad \text { a.e. in } \Lambda
$$


where $\theta_{n} \in \mathbb{R}, \tilde{\psi}_{n}$ solves (7.39) with $\varrho_{n}$ replaced by $\tilde{\varrho}_{n}$ and

$$
\varepsilon_{n}=\varepsilon_{n}^{1}+\varepsilon_{n}^{2}, \quad \varepsilon_{n}^{1} \rightarrow 0 \text { in } L_{\infty}(\Lambda), \quad \varepsilon_{n}^{2} \rightarrow 0 \text { in } H_{0}^{1}(\Lambda) .
$$

Observe also that multiplying (7.39) by $\tilde{\varrho}_{n}$ and using the fact that $\tilde{\varrho}_{n}$ is a maximizing sequence of $I_{8 \pi}(\Lambda)$ we obtain

$$
\left.\left|\theta_{n}-\frac{1}{2} \int\right| \nabla \tilde{\psi}_{n}\right|^{2} \mid \leqq I_{8 \pi}(\Lambda)+\delta_{n}+C\left\|\varepsilon_{n}^{2}\right\|_{H_{0}^{1}}\left\|\nabla \tilde{\psi}_{n}\right\|_{L_{2}},
$$

where $\delta_{n} \underset{n}{\longrightarrow} 0$ and $C$ is some positive constant not depending on $n$.

Next, since $\varrho_{n}-\tilde{\varrho}_{n} \stackrel{n}{\longrightarrow} 0$ in $H^{-1}(\Lambda)$, we deduce first that

$$
\int_{\Lambda}\left|\nabla \tilde{\psi}_{n}\right|^{2} \underset{n}{\longrightarrow} \infty
$$

and thus $\int_{\Lambda} \tilde{\varrho}_{n} \log \tilde{\varrho}_{n} \underset{n}{\longrightarrow} \infty$ because $\tilde{\varrho}_{n}$ is maximizing. Finally,

$$
\left|\nabla \psi_{n}\right|^{2}\left(\int_{\Lambda}\left|\nabla \psi_{n}\right|^{2}\right)^{-1}-\left|\nabla \psi_{n}\right|^{2}\left(\int_{\Lambda}\left|\nabla \tilde{\psi}_{n}\right|^{2}\right)^{-1} \stackrel{n}{\longrightarrow} 0 \quad \text { in } L_{1}(\Lambda),
$$

and $\left(\int\left|\nabla \psi_{n}\right|^{2}\right)\left(\int\left|\nabla \psi_{n}\right|^{2}\right)^{-1} \rightarrow 1$. Therefore, using once more the fact that $\varrho_{n}$ and $\tilde{\varrho}_{n}$ are both maximizing we also deduce that $\left(\int_{\Lambda} \varrho_{n} \log \varrho_{n}\right)^{-1} \cdot\left(\int_{\Lambda} \tilde{\varrho}_{n} \log \tilde{\varrho}_{n}\right) \underset{n}{\longrightarrow} 1$.

All the above information allows us to extend Step 2 to a general unbounded maximizing sequence. Summarizing, we have proved that if the equality sign occurs in (7.20) then there is an unbounded maximizing sequence (Step 1). On the other hand any maximizing unbounded sequence leads to the equality in (7.20) and has the behavior described in claim 2) (Step 3). The last statement in claim 2) follows easily by the previous considerations.

As we mentioned above, although we are not able to solve the alternative even for domains close to a disk, we can exhibit an example of convex domains in which the concentration does not occur. Consider for example a rectangle with sides $a$ and $b$. By virtue of Theorem 7.1. If the concentration takes place, we would have

$$
I_{8 \pi}(\Lambda)=I_{8 \pi}\left(\Lambda_{0}\right)+\gamma\left(x_{0}\right)-\frac{1}{8 \pi} \log \frac{(a \cdot b)}{\pi} .
$$

Taking $b$ sufficiently large the above expression can be made negative, since $\gamma\left(x_{0}\right)$ stays bounded from above. On the other hand $\bar{g}(\psi=0)=0$ and this contradicts the concentration. It has to be noticed that on the basis of Proposition 7.1, if there exist solutions of the mean field equation (necessarily not maximizing by Proposition 7.3) they must disappear for sufficiently large negative $\beta$. We can give many more examples of "non-concentration" phenomena for instance for thin annuli.

We conclude the section by discussing the stability properties of the solutions of the mean field equation thought of as stationary solutions of the 2-D Euler equation. Following Arnold $[3,4]$, we consider the ratio:

$$
\frac{\nabla \varrho}{\nabla \psi}=-\frac{\beta e^{-\beta \psi}}{\int e^{-\beta \psi}} \text {. }
$$

If $\beta>0$, by virtue of the first Arnold's Theorem the solution of the mean field equation is stable with respect to the norm $\frac{1}{2} \int|\nabla \psi|^{2}+|\Delta \psi|^{2}$. If $\beta<0$ we can apply 
the second Arnold's theorem ensuring the stability if

$$
\inf _{x \in \Lambda} \frac{e^{\beta \psi} \int e^{-\beta \psi}}{|\beta|}>\sup _{\varphi} \frac{\int|\nabla \varphi|^{2}}{\int|\Delta \varphi|^{2}}
$$

which is certainly fulfilled if $|\beta|$ is small.

We do not know whether the solutions for negative $\beta$ close to $-8 \pi$ are unstable. However, for the particular cases of the symmetric solutions studied in Sect. 5, we can use the results of Marchioro and Pulvirenti [30] in order to prove the stability (in the norm $\left.\int|\Delta \psi|\right)$ for all $\beta \in(-8 \pi,+\infty)$.

Note added in proof. After final submission of the paper, the following reference was discovered which is also relevant to our subject: Lundgren, T. S., Pointing, Y. B.: J. Stat. Phys. 17, 323-355 (1977)

\section{References}

1. Albeverio, S., Hoegh-Krohn, R.: Phys. Rep. 20, 585-595 (1979)

2. Albeverio, S., Hoegh-Krohn, R., Merlini, D.: Some remarks on Euler flows associated to generalized random fields and Coulomb systems. Bibos Preprint, 1985

3. Arnold, V.I.: Dokl. Nat. Nauk. 162, 773-777 (1965)

4. Arnold, V.I.: Am. Math. Soc. Transl. 79, 267-269 (1969)

5. Bahri, A., Coron, J.M.: C.R. Acad. Sci. Paris (1985)

6. Benfatto, G., Picco, P., Pulvirenti, M.: J. Stat. Phys. 46, 729 (1987)

7. Benzi, R., Patarnello, S., Santangelo, P.: Europhys. Lett. 3, 811-818 (1987)

8. Benzi, R., Patarnello, S., Santangelo, P.: J. Phys. A 21, 1221-1237 (1988)

9. Boldrighini, C., Frigio, S.: Att. Sem. Mat. Fis. Univ. Modena 27, 106-125 (1978), and Commun. Math. Phys. 72, 55-76 (1980)

10. Brézis, H., Peletier, L.A.: Asymptotics for elliptic equations involving critical growth. In: Partial differential equations and the calculus of variations, Vol. I. Basel: Birkhäuser 1989

11. Caprino, S., De Gregorio, S.: Math. Meth. Appl. Sci. 7, 55-70 (1985)

12. Chang, S.Y.A., Yang, P.: Acta Math. 159 (1988)

13. Chang, S.Y.A., Yang, P.: J. Diff. Geom. 23 (1988)

14. Chen, W., Ding, W.: Trans. A.M.S. 303 (1987)

15. DiPerna, R.J., Majda, A.: Commun. Math. Phys. 108, 667-689 (1987)

16. DiPerna, R.J., Majda, A.: Commun. Pure Appl. Math. 40, 301-345 (1987)

17. Ekeland, I.: Bull. A.M.S. 1, 443-474 (1979)

18. Esteban, M.J., Lions, P.L.: Proc. Roy. Soc. Edin. 83, 1-14 (1982)

19. Fröhlich, J., Ruelle, D.: Commun. Math. Phys. 87, 1-36 (1982)

20. Gallavotti, G.: Problèmes ergodiques de la mécanique classique. Cours de 3e cycle, Physique, E.P.F. Lausanne (1976)

21. Gidas, B., Ni, W.M., Nirenberg, L.: Commun. Math. Phys. 68, 203-243 (1979)

22. Gogny, D., Lions, P.L.: RAIRO Modél. Math. Anal. Num. 23, 137-153 (1989)

23. Gustaffsson, B.: On the motion of a vortex in two-dimensional flow of an ideal fluid in simply and multiply connected domains. Tech. Rep. Dep. of Math., Royal Inst. of Technology, Stockholm (1979)

24. Haegi, H.R.: Compositio Math. 8, 81-111 (1951)

25. Han, Z.C.: On some nonlinear ordinary and partial differential equations. Ph.D. thesis, NewYork University, 1989

26. Hong, C.: Proc. A.M.S. 87 (1986)

27. Hopf, E.: J. Rat. Mech. Anal. 1, 87-123 (1952)

28. Lions, P.L.: Rev. Mat. Iberoamericana 1, 145-201 (1985)

29. Lions, P.L.: Commun. Math. Phys. 109, 33-57 (1987)

30. Marchioro, C., Pulvirenti, M.: Commun. Math. Phys. 100, 343-354 (1985)

31. Messer, J., Spohn, H.: J. Stat. Phys. 29, 561-578 (1982)

32. Moser, J.: Indiana Univ. Math. J. 20, 1077-1092 (1971) 
33. Onofri, E.: Commun. Math. Phys. 86, 321-326 (1982)

34. Onsager, L.: Suppl. Nuovo Cim. 6, 279 (1949)

35. Pohozaev, S.I.: Sov. Math. Dokl. 6, 1408-1411 (1965)

36. Pulvirenti, M.: On invariant measures for the 2-D Euler flow. In: Mathematical aspects of vortex dynamics. Caflisch, R. (ed.) SIAM Philadelphia (1989)

37. Rey, O.: J. Fund, Anal. 89, 1-52 (1990)

38. Ruelle, D.: Statıstical mechanics. New York: Benjamin 1969

39. Sacks, J., Uhlenbeck, K.: Ann. Math. 113, 1-24 (1981)

40. Struwe, M.: Math. Z. 187, 511-517 (1984)

Communicated by J. L. Lebowitz 
